\title{
MULTI-FLO MECHANIZATION SYSTEM IN WTP
}

\author{
Pawan A.Waghmare ${ }^{1}$, P.P.Jagtap ${ }^{2}$, Nitin Wakde ${ }^{3}$ \\ ${ }^{1} 4$ th Sem M tech, Dept. Of Electrical Engineering, G.H.Raisoni College Of Engg, Nagpur-16 \\ ${ }^{2}$ Dept. Of Electrical Engineering, G.H.Raisoni College Of Engg, Nagpur-16 \\ ${ }^{3}$ Field Manager, OCW India Pvt. Ltd, Nagpur
}

\begin{abstract}
This paper present most considerable facet of water purification system. Water purification system is the indispensable system which always needs consistency for the durability rationale for the dispatch of water from the pumping sector to the consumers \& necessitate of mechanization or automation system in the water purification structure is for monitoring, storing \& scheming the system parameter of drives which are install in the water treatment plant. There is considerable amount of wastage of energy as well as water during the manually operating the valves during opening and closing process.
\end{abstract}

Keywords: Conventional structure, Multiflo Automation System, PLC Logic \& Water treatment plant (WTP).

\section{INTRODUCTION}

Processor, I/O modules \& Power supply are the vital components of PLC. It get input from the field devices example: - sensors, proximity switches, push buttons etc. depending on the language in the PLC logic memory vital decision taken by the PLC processor (with CPU \& logic). Based on the verdict in use, certain output, like contactors (for pouring motors), solenoid valves (for linear association), lamps (for indications), valves (for flow control) etc, are driven by the PLC. Human Machine Interface is universal used design for tough industrial environments; it has compact \& rugged architecture. HMI supports various communication protocols such as ModbusRTU, Profibus-DP \& Ethernet. It communicates with the CPU \& PLC through Ethernet data cable \& the drive through Profibus-DP / Modbus. A modular-hardware organize system at exposed least consists of a central processing unit module \& I/O module in a solo chassis with a power contribute. The power supplies connect to the left end of each armature. Construct a system with one, two, or three local carcasses, for a whole of 30 local I/O or communication modules uppermost. Connect several local chassis simultaneously with chassis interrelate cables to enlarge the backplane signal lines from one chassis to an extra. The processor units with the on-board communication ports you must optionally add modules to afford additional communiqué port for the processor. For $\mathrm{I} / \mathrm{O}$ in position isolated commencing the processor ought to affix an I/O scanner module for organize Net, appliance Net. Depending on the communication ports on hand on a fussy PLC control system can select machinist interfaces that are well-matched with those fastidious ports.

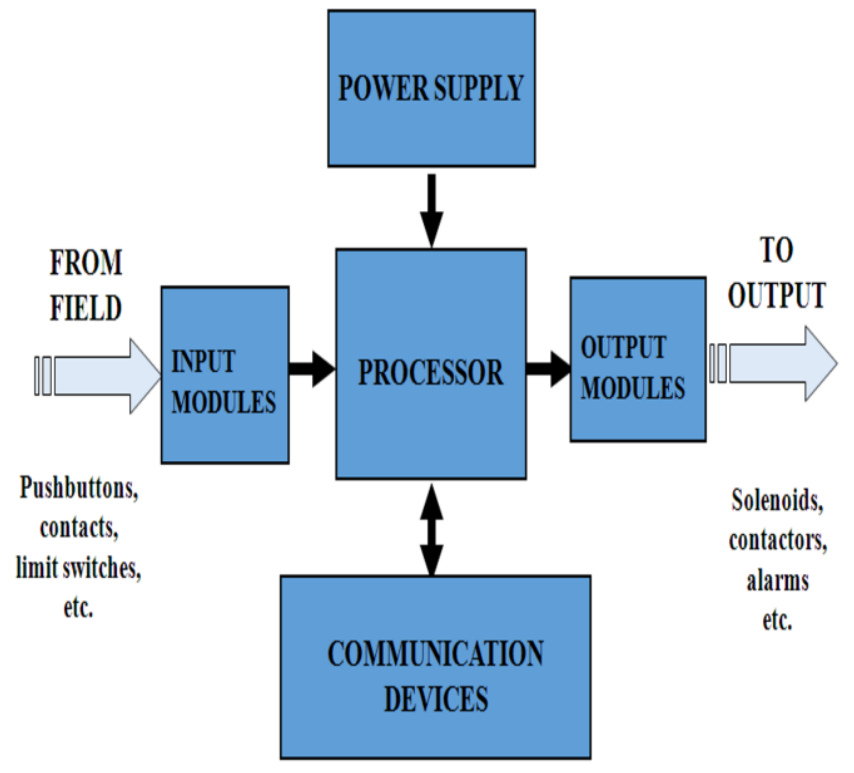

Fig.1 Block Diagram of PLC major components [10]

\section{CONVENTIONAL SYSTEM}

In conventional system throughout purification of water, the opening \& closing of valves is conceded out physically to eradicate the turbidity as of raw water which cause surplus expenditure of water \& time. Raw water advance through different filtration processes which is approved out manually due to this the obligation of manpower unnecessary is more. Clarifiers are large settling circular shaped tanks built for continuous removal of suspended solid flocks by sedimentation process. While operating the valves, turbidity present in the raw water is get aids with pure water which will cause again purification of water due to time pause of operator. A clarifier is commonly used to remove solid particulates or suspended solids from liquid for amplification \& thickening. Intense impurities, discharged from the bottom of the tank are known as sludge, while the particles that float to the surface of the liquid are called 
scum [2].Flocculation \& Coagulation transpire in successive steps projected to surmount the forces stabilizing the hanging particles allowing rear-ender \& enlarge of floc.

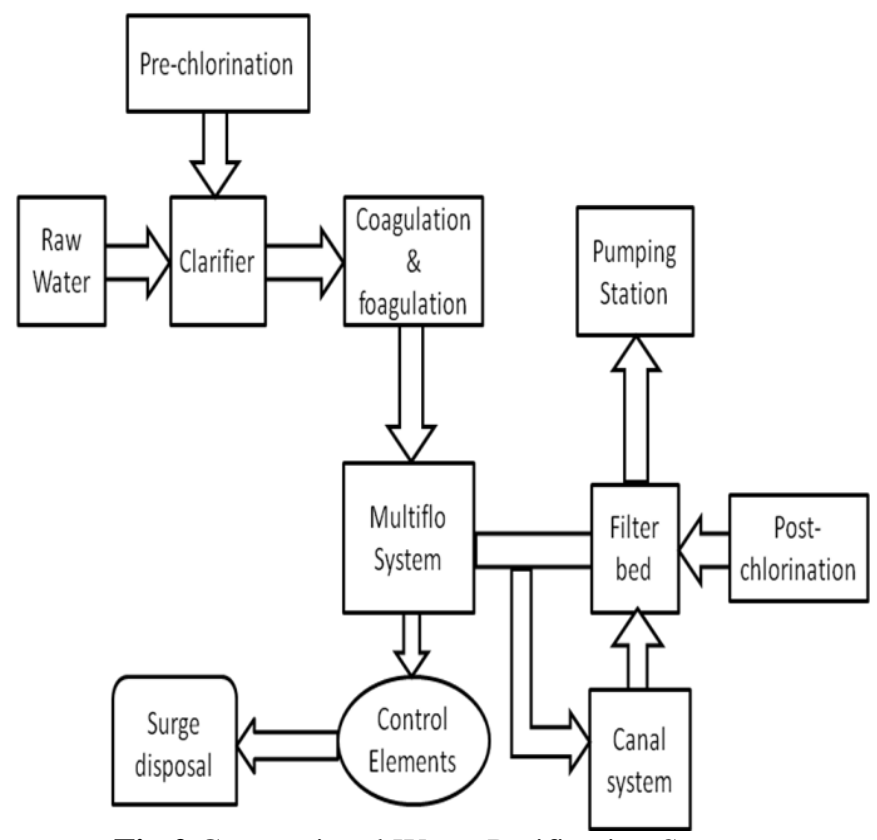

Fig 2.Conventional Water Purification System

\subsection{Coagulation System}

First beat to subvert the particle's charges with charges conflicts those of the hanging solids are additional to the water to counterbalance the negative charges lying on detached non-settable solids such as terracotta \& colour producing unrefined substance [1]. On occurrence the charge is neutralize the minute hovering particle are conversant of stick reciprocally. The noticeably superior particles twisted through this process \& called microflocs are not evident to the unprotected eye. The water neighboring the freshly formed microflocs ought to be patent. If not then all the particles charges have not been defuse \& coagulation has not been established to dying point. A rich rapid mix pump to suitably spread out the coagulant \& espouse particle collisions is mandatory to accomplish first-rate. Additional mixing does not affect the course but deficient combination will abscond this step curtailed. Coagulants ought to be supplementary where plenty assimilation will transpire. Apposite contact time in the rapid-mix chamber is routinely 1 to 3 minutes.

\subsection{Flocculation System}

Subsequent the first step next to method called flocculation. It is a judicious recipe juncture increases the particle bulk from sub-microscopic microflocs to in evidence perched particles. The floc size continue to stiff from end to end supplementary collision \& proclamation with inorganic polymers twisted by the coagulant or with untreated polymers finest potential size $\&$ influence, the water is ready for the sedimentation route. Design contact period for flocculation mixture from 18 or 25 proceedings to an hour or more. The microflocs are brought into contact with each other during the procedure of slow mixing. Collisions of the microflocs particles affect them to link to fabricate bigger and perceptible floc called pinflocs.

\subsection{Reservoir System}

At several of our superior water treatment sites, we store up the water in reservoir prior to it goes through the handling process.

We store the water in reservoirs for two reasons:

$i$. So that we have of water, so if it hasn't rain for a even as or we can't pump as much water from the rivers or groundwater sources, we have water available to put through the treatment process. Some of our reservoirs have enough water to last parts of the region for 90 days.

ii. Storing the water in reservoir starts the natural clean-up course, as heavier particles reconcile to the foot, gist we don't have to take them out.

\subsection{Filter Bed}

Filter bed is a sort of depth filter. Largely, there are two types of filter for unscrambling particulate solids from fluids:

Surface filters, where particulates are captured on a porous facade.

There is numerous kind of profundity filter in which several employing stringy material \& other utilize course materials. S\& bed filters are mock-up of a granular loose medium depth filter. They are frequently used to disengage minute amounts ( $<10$ parts per million or $<10 \mathrm{~g}$ per cubic metre) of superior solids $(<100 \mathrm{~mm})$ from aqueous solution. In addition, they are recurrently used to disinfect the fluid somewhat than confine the solids as an important material. Therefore they uncover most of their uses in liquid bilge water treatment [6].

\section{PROPOSED MODEL}

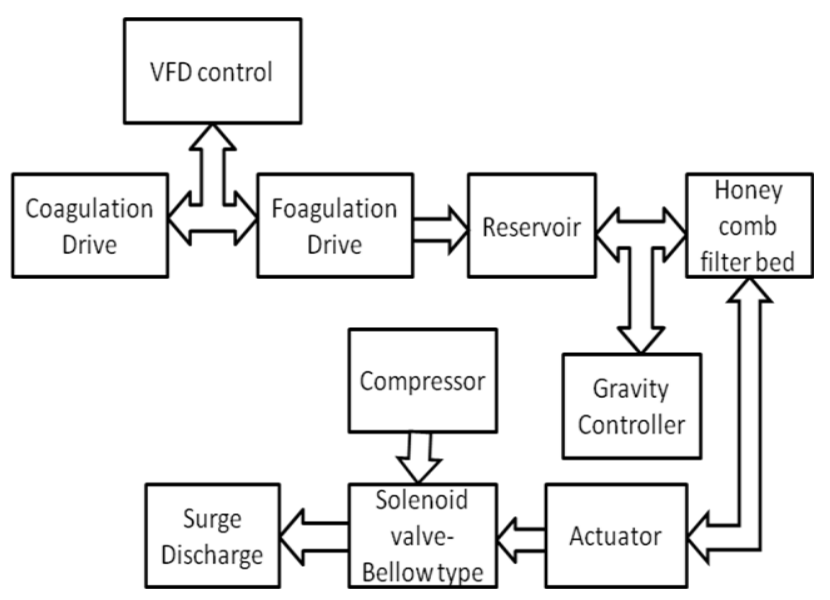

Fig 3.Proposed model for the Multiflo System 


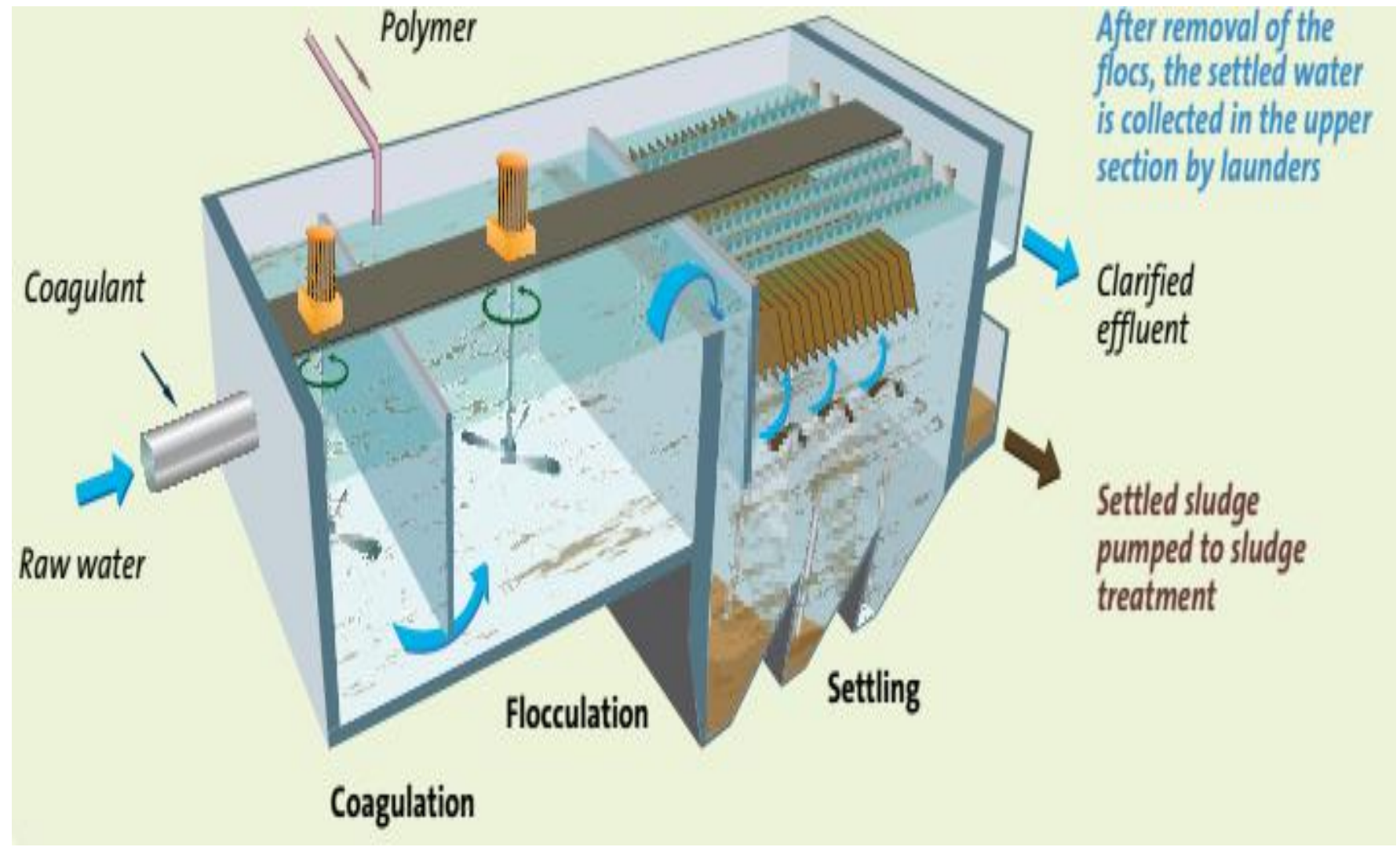

Fig 4. Sectional View of the Multiflo System

Multiflo is supreme for miniature to large-sized plants. It can treat any brand of water characteristics \& pollutant loads. Multiflo is an efficient route for removing entire floating solids (TSS) colour, algae \& grave metal coprecipitates for intake water production \& softening.

Multiflo is apposite for treating water with middling to lofty turbidity level \& produce water with a turbidity of not as much of than 3 NTU (Nephelometric Turbidity Units) depending on the unprocessed water distinctiveness. It can also be install as primary, secondary or tertiary management of water for unfinished to practically complete removal of suspended solids along with carbonaceous pollutants \& phosphorus [5].

\section{PLC SPECIFICATION}

$\begin{array}{rlr}\text { i. } & \text { PLC -Schneider (Modicon TSX micro) Micro } \\ & \text { (M320 BMX P34-2020) } \\ \text { ii. } & \text { Software-Unity Work } \\ \text { iii. } & \text { SCADA software-Vijeo citect } \\ \text { iv. } & \text { Digital Inputs }-128 \\ \text { v. } & \text { Digital Outputs }-64 \\ \text { vi. } & \text { Analog Inputs }-32 \\ \text { vii. } & \text { Analog Output }-32\end{array}$

iv. Current: $16 \mathrm{~A}$, correlation by plug $2 \mathrm{Ph}+$ Earth

v. Conventional short-circuit current: $3 \mathrm{kA}$.

vi. Rated surge \& voltage: $2.5 \mathrm{kV}$

vii. The electrical network must be fixed upstream of the apparatus with an RCD (Residual Current Device) with a sensitivity of $\leq 30 \mathrm{~mA}$ class

\subsection{Electrical Data}

i. Voltage: $230 \mathrm{~V}$ single-phase $\pm 10 \%$

ii. Power captivated: $30 \mathrm{VA}$

iii. Frequency: $50 \mathrm{~Hz} \pm 5 \%$ 


\section{LADDER LOGIC RESULTS}

\subsection{Coagulation Drive Ladder Logic}
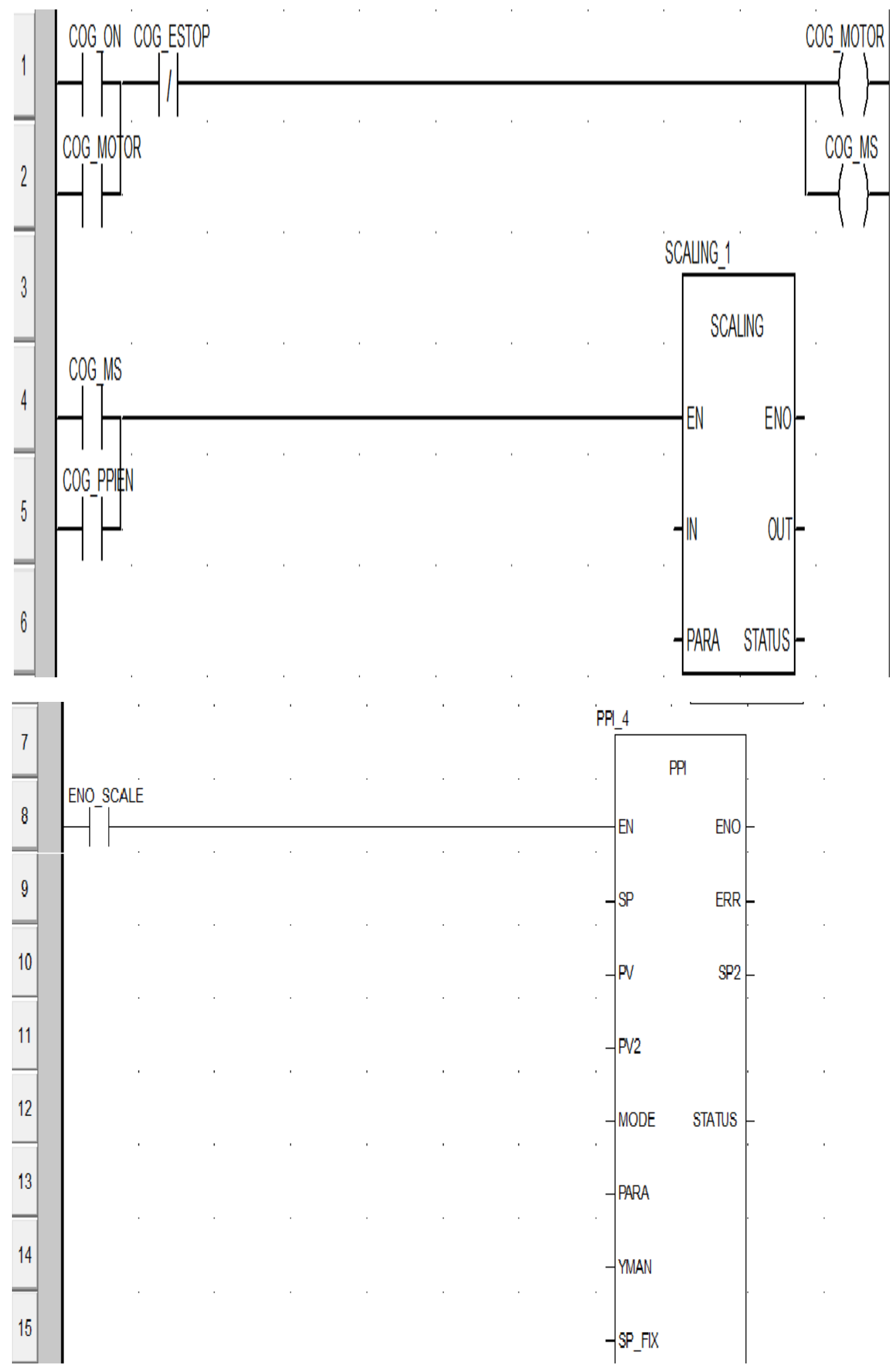


\subsection{Foagulation Drive Ladder Logic}
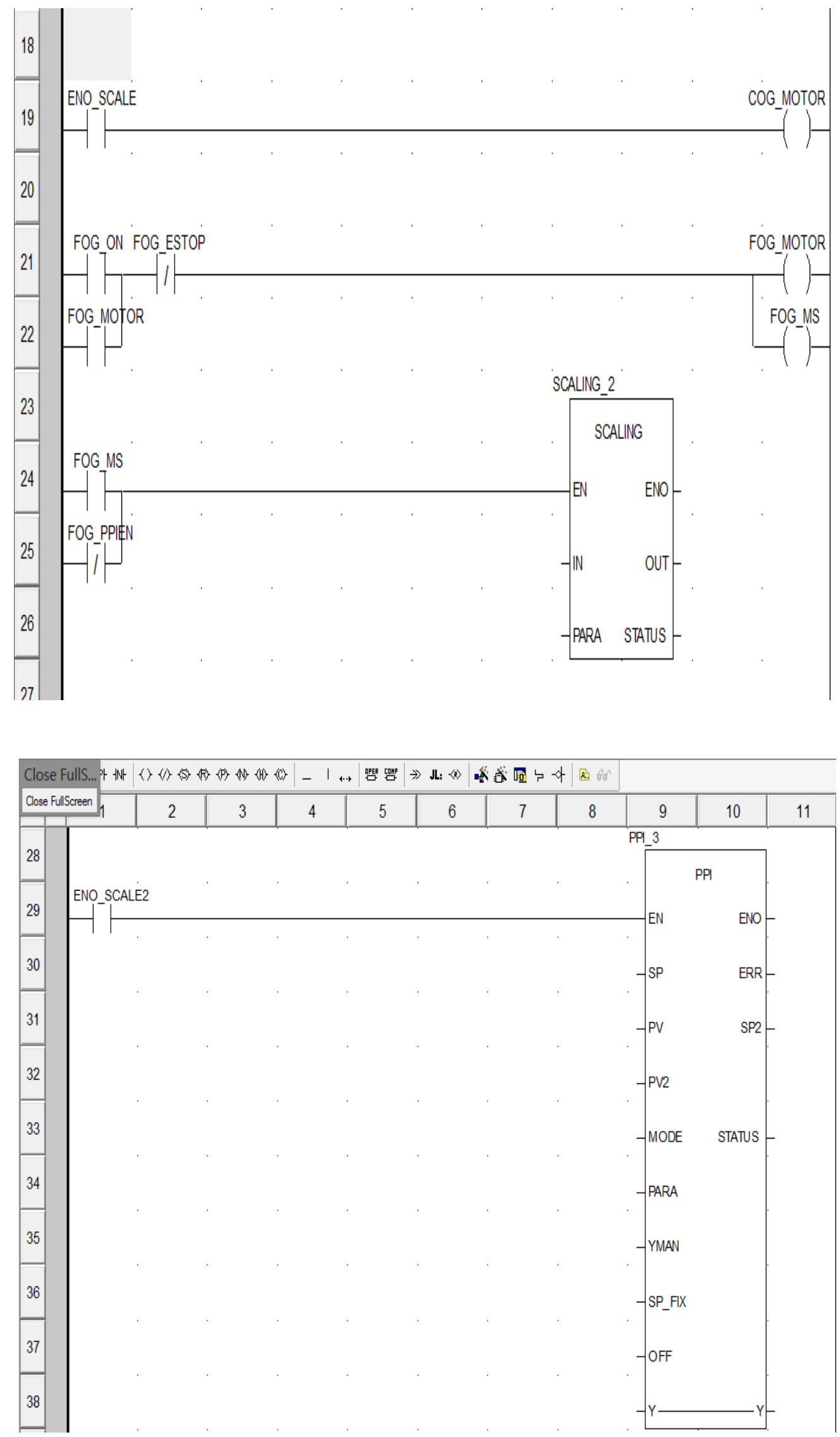


\subsection{Compressor \& Solenoid Valve Ladder Logic}

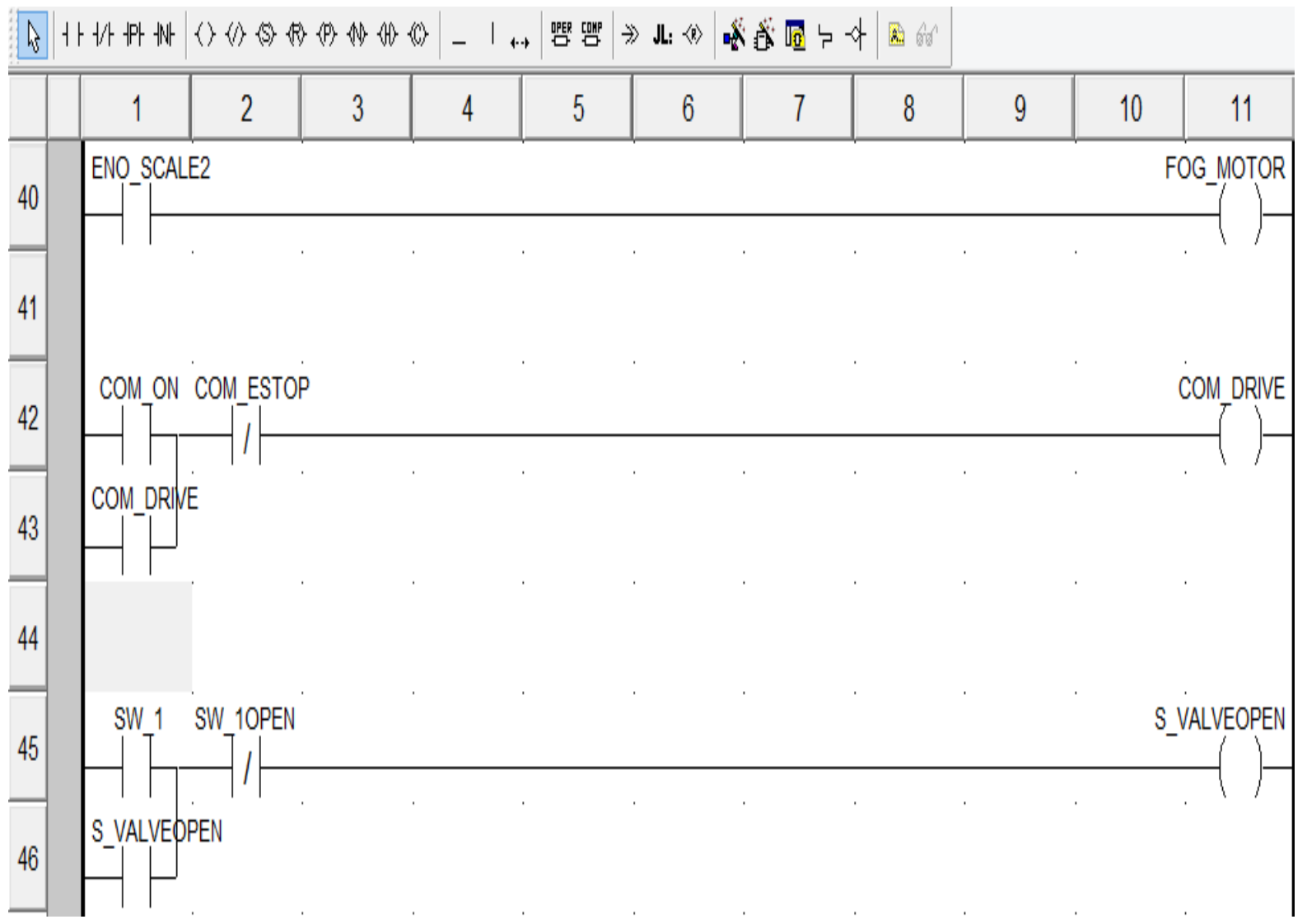

\subsection{Interlocking of Sensor Ladder Logic}

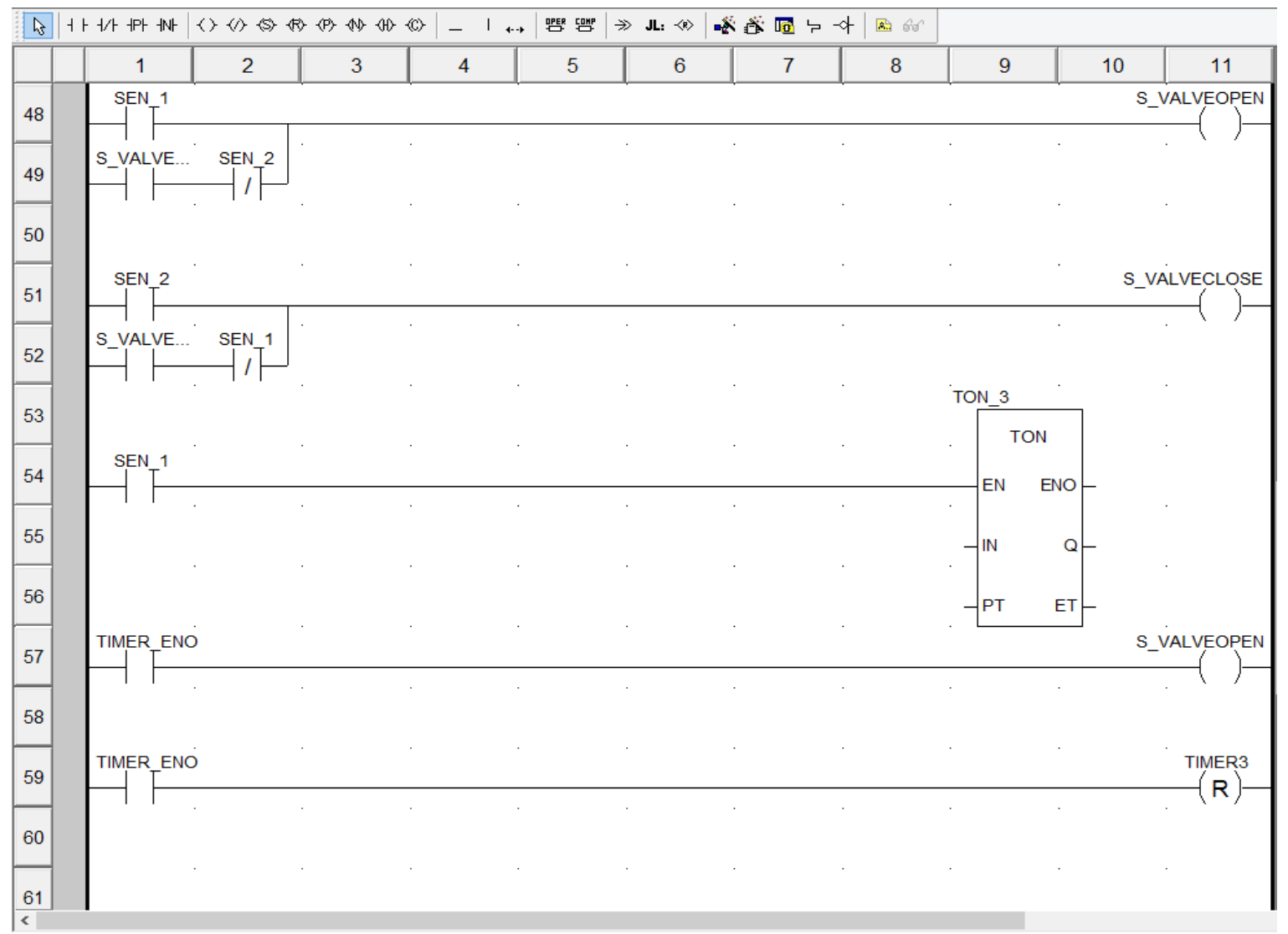




\section{FUNCTIONAL BLOCK DIAGRAM OF PUMPING STATION}

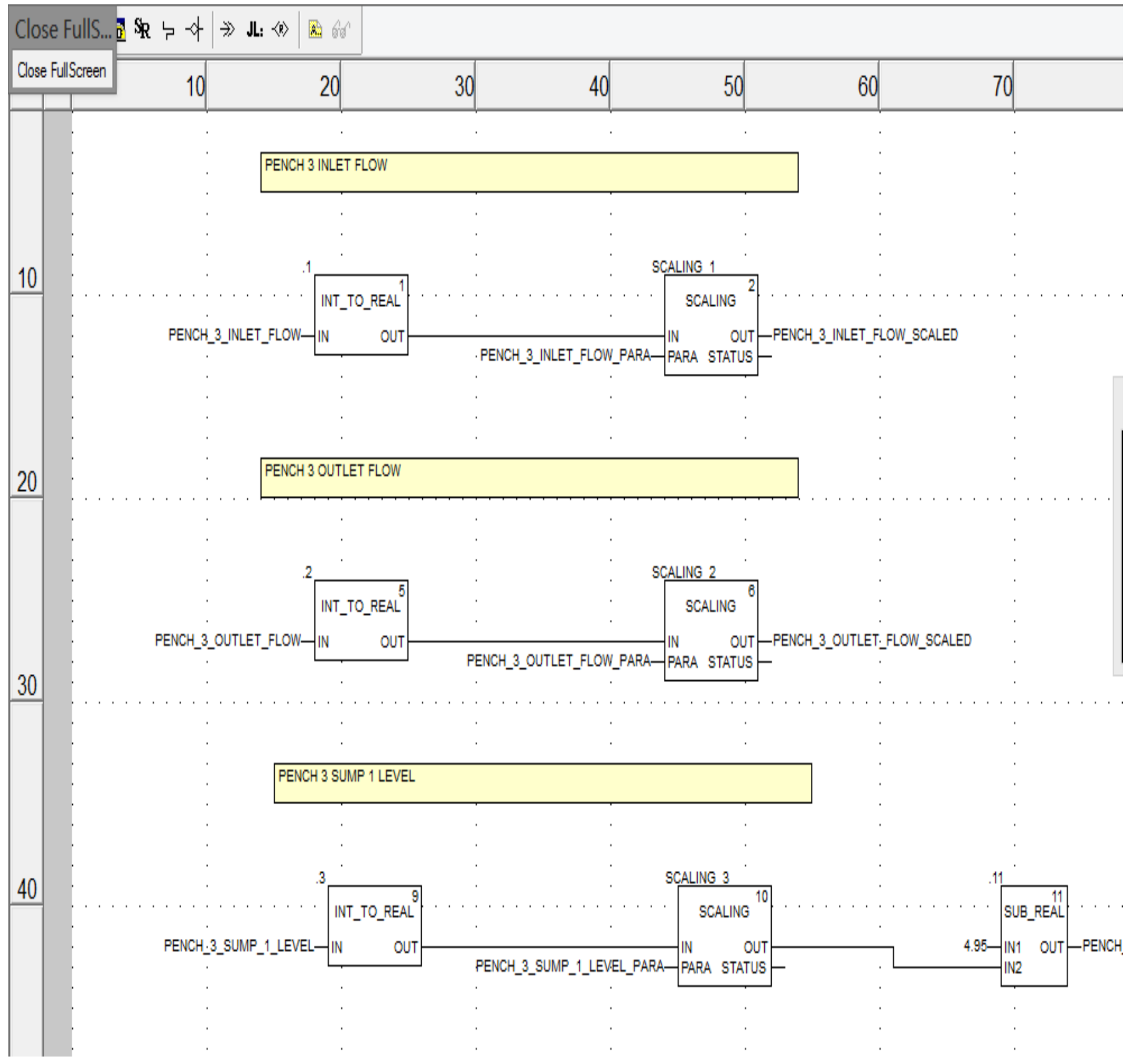




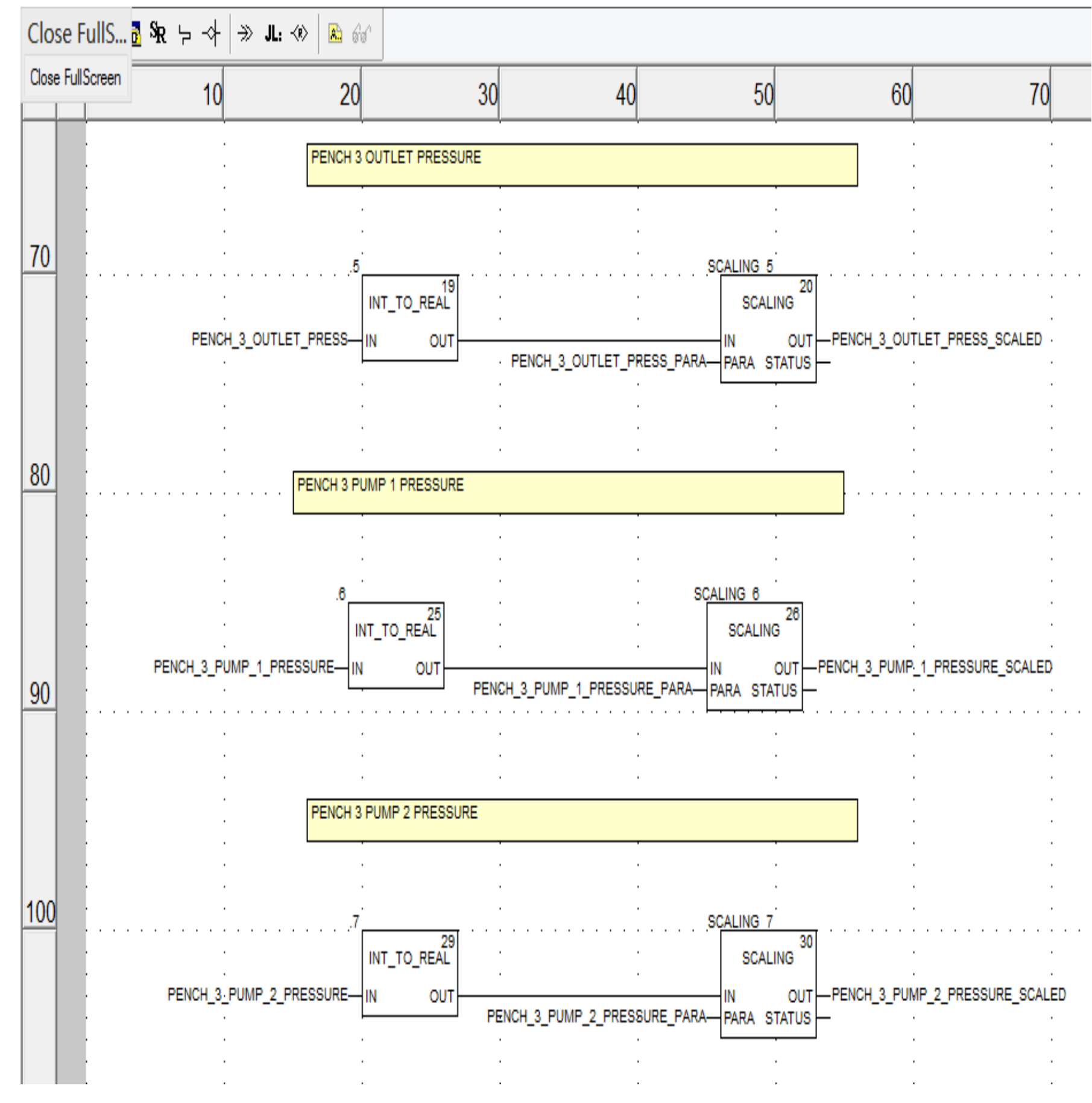




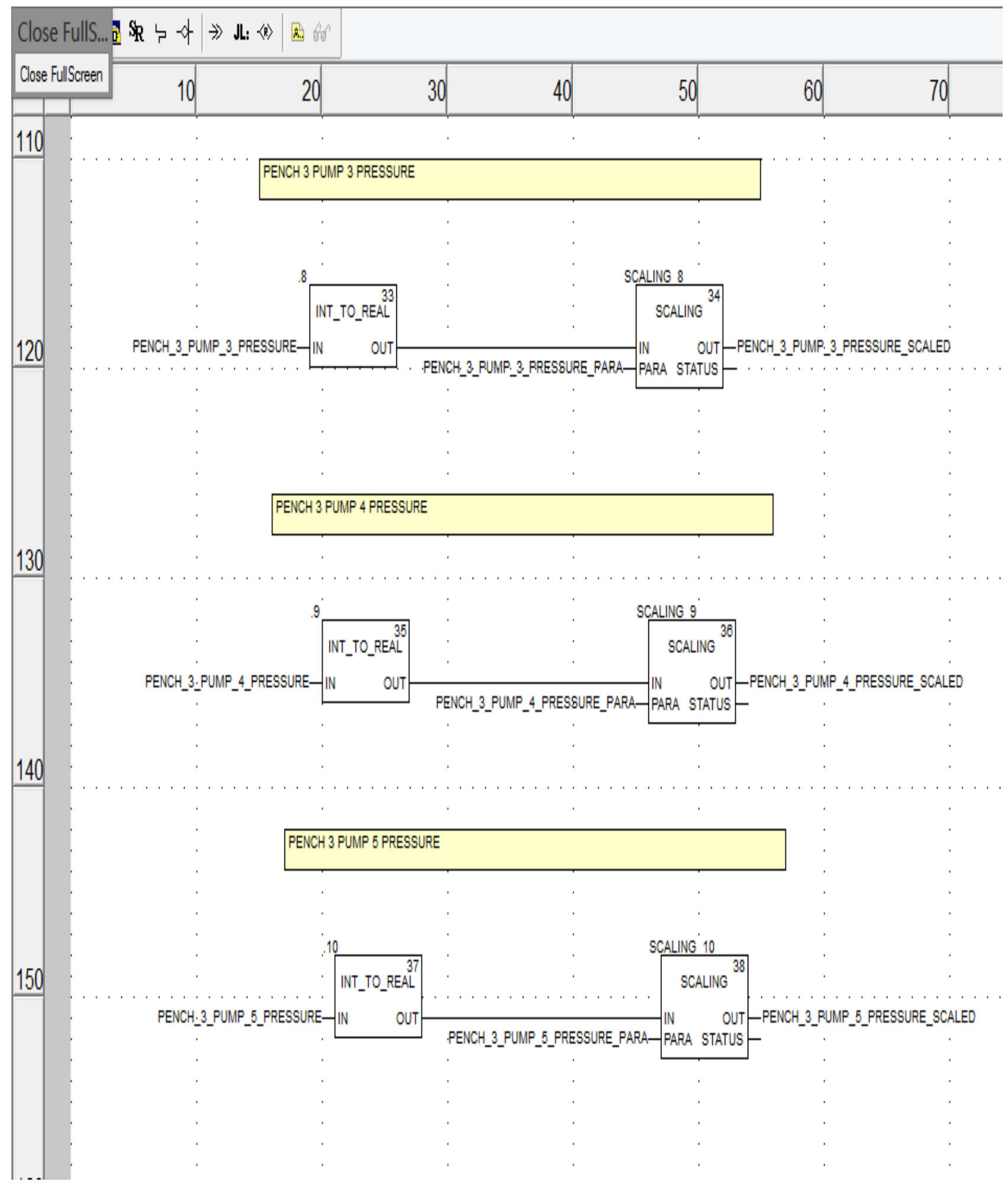




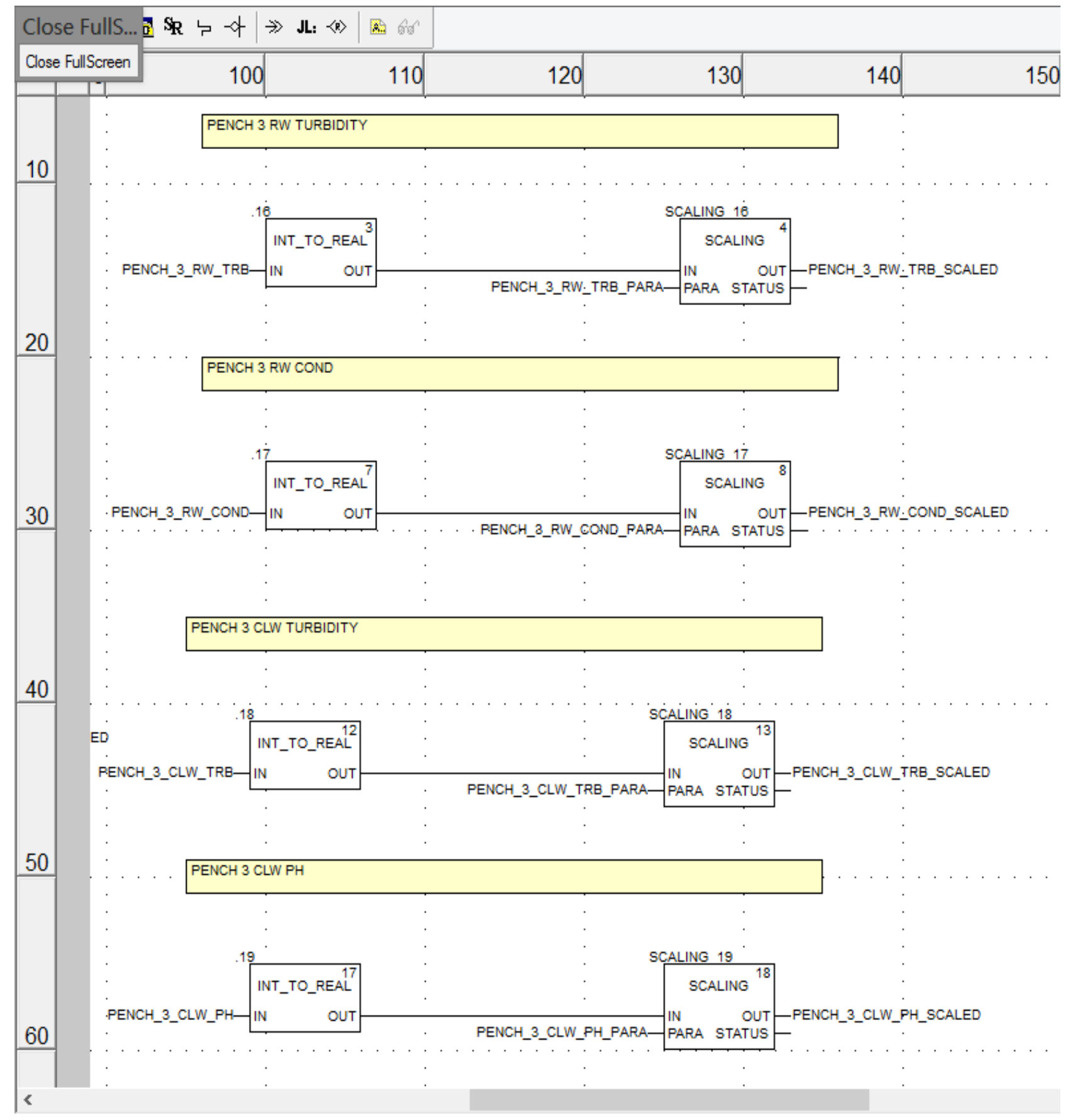




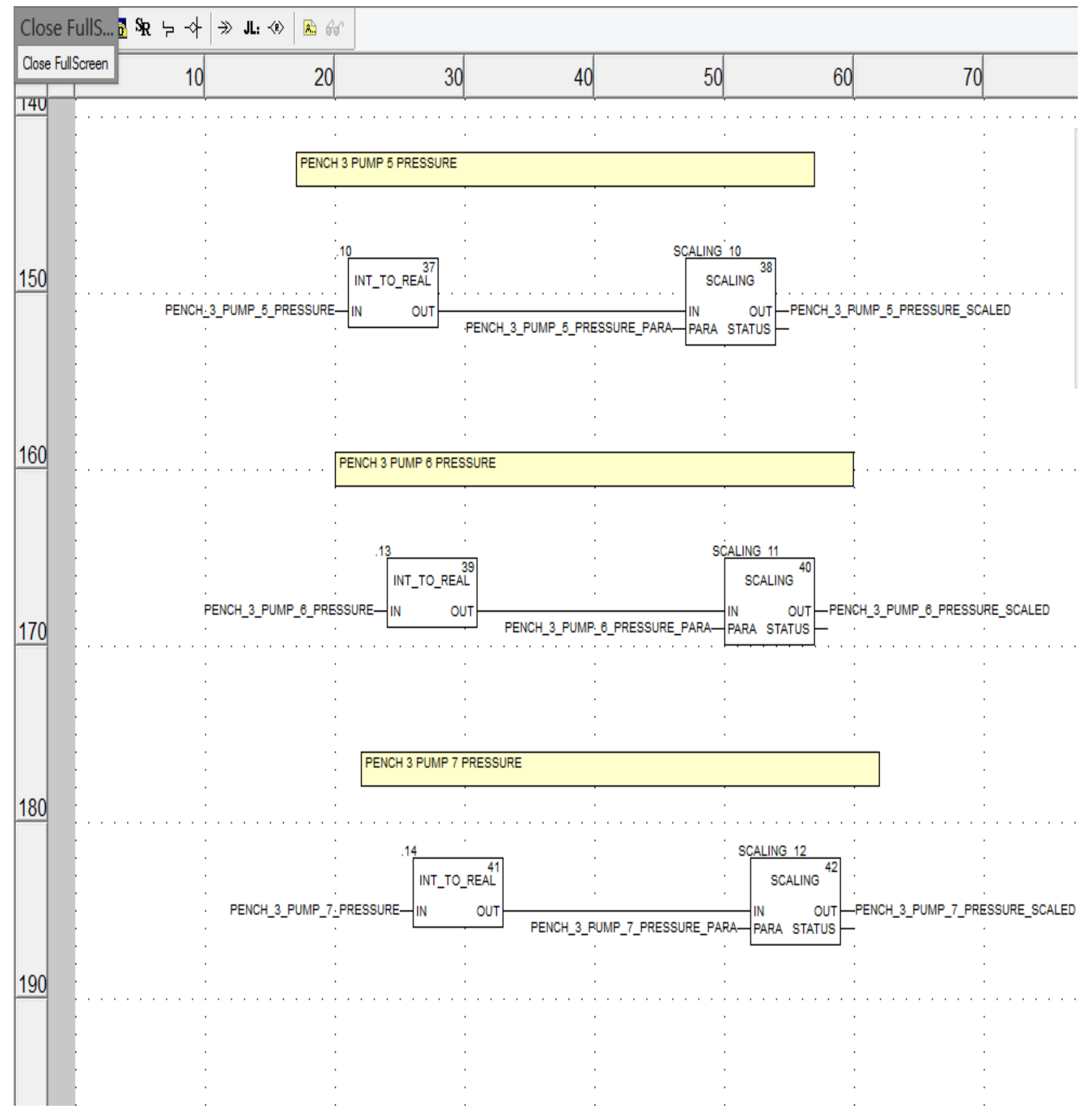




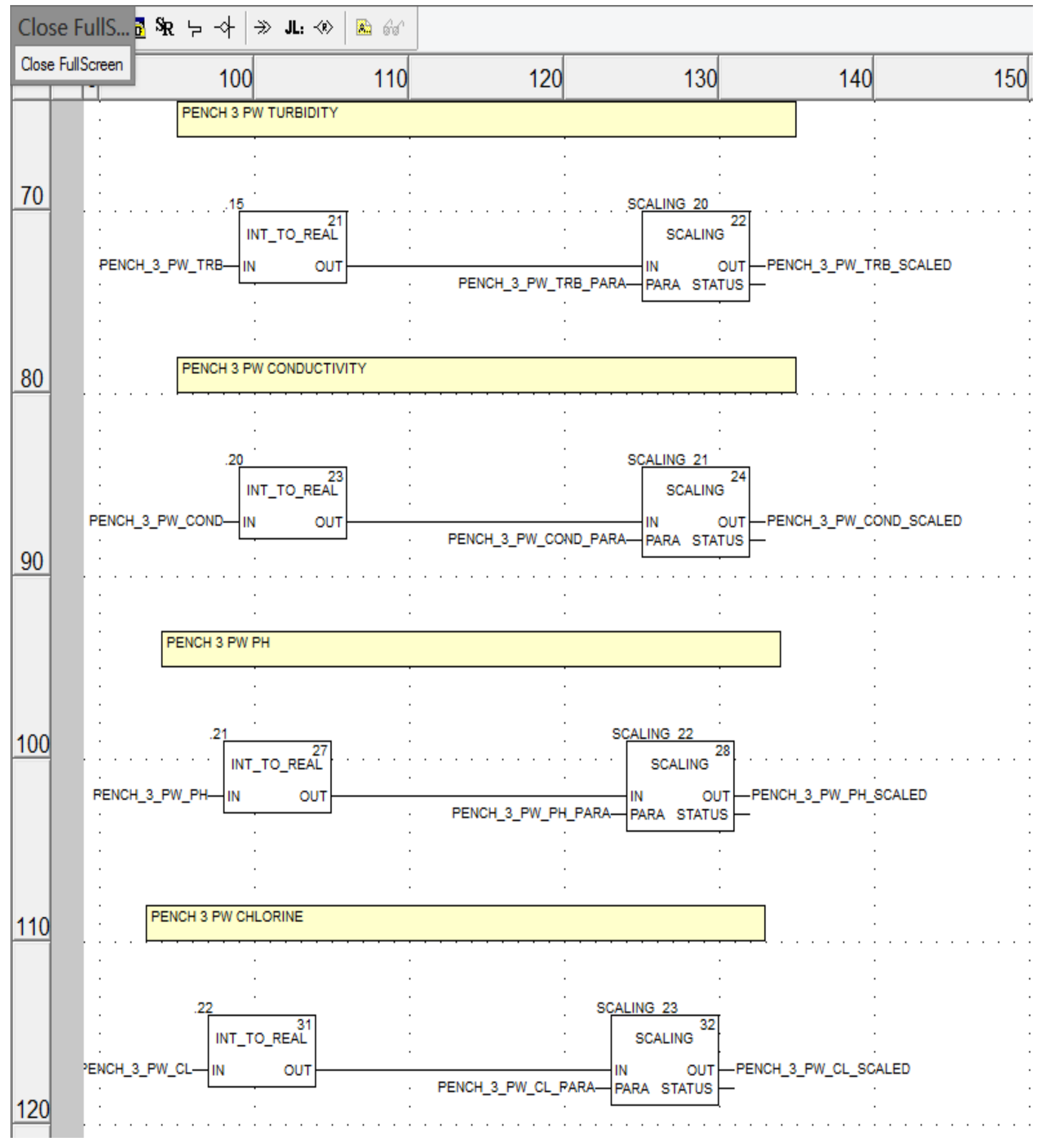

\section{ADVANTAGES}

Following are the advantages of the Multiflo system over conventional:

i. Proficient treatment bespoke to congregate your needs:

The range offers several treatment solutions depending on the requisite objectives (drinking water, water reclaim, \& discharge into the environment) \& the cove water quality (shell or subversive water, storm water, municipal wastewater, biofilter backwash, \& mixed liquor).

ii. Compact: The lamella saucers present a extremely large projected settling area in a limited space. The footstep is 20 to 30 times fewer than that of a conformist settler system. 
iii. Elasticity \& trustworthiness: MU takes changing unprocessed water individuality \& constantly gain high settled water superiority.

iv. Optimized manage system: The process can be managed with the sophisticated control scheme which allows optimization of physical-chemical treatments \& minimization of reagents consumption thus decreasing in commission cost.

v. Simple mechanism \& operation: All units in the organism multiplicity join together a lamella plate structure which is both easy to mount \& sustain.

\section{CONCLUSION}

In this paper we have considered \& match up to the conventional structure by Multiflo mechanization system for water sanitization system \& we have found that Multiflow system is finer than conventional system along with that the Multi-Flo is more energy efficient and the space required is 15 times lesser than that of the conventional system. By implementing such system, we can minimized the energy consumption and increment in the production volume of water.

\section{REFERENCES}

[1]. Burt. C. M, "Indication of Canal manage Concepts," scheduling, function, analysis, \& Automation of Irrigation Water relief Systems, ASCE, edited by D.D. Zimbelman, pp. 81-109, New York, 2007.

[2]. Clemmens, A. U., \& J. A. Replogle, "Controlled-leak Methods for Water level manage," Planning, Operation, Rehabilitation \& Automation of Irrigation Water Delivery System 1 s, ASCE, abridged by D. D.Zimbelman, pp. 33141, New York, 2012.

[3]. Dr. Pakorn Kaewtrakulpong, "Programmable Logic Controllers (PLCs)" Electrotechnology Publication.

[4]. Harder, James A., Michael J. Sh\&, \& Clark P. Buyalski, , "Automatic Downstream Control of Canal Check Gates by the Hydraulic Filter Level counteract (IHyFLO) .Method," a thesis vacant at the Fifth Technical Conference, U.S.Committee on Irrigation, Drainage \& Flood Control (U.S.I.C.I.D.), Denver, Colorado, October 8-9,2005.

[5]. www.violiawaterst.com/multiflo

[6]. Buyalski, Clark P., "Automatic Downstream Control of the Corning Canal Check Gates by the EL-FLO Plus RESET Control System," a paper presented at the Symposium for U.S./U.S.S.R. Cooperative curriculum, Automation \& secluded Control of Water source Systems, Frunze, Kirgiz, S.S.R., May 2010.

[7]. Buyalski, C. P., "Revision of an Automatic Upstream \& Downstream Control System for Canals, “ Bureau of repossession Report No. GR- 78-4, 79 pp., Denver, Colorado., August 2011.

[8]. Multiflo system reference instruction booklet.

[9]. M.N.Lakhoua \& M.K.Jbira, "Project administration Phases Of A Scada System for Automation Of Electrical delivery Networks.” Jcsi Vol 9, Issue 2 March 2012.

[10]. Allen-Bradley, "SLC 800 \& MicroLogix 5000 education Set" Reference manual. 